you could not, and hit the stone without probably you could not; while, if the unfitness of the instrument for the special case had not crossed your mind, it would probably be withdrawn without finding anything, and you would treat the patient for something else till he had grown tired of his non-improvement and had sought the advice of some abler man. Many a stone has been missed behind the prostate simply because the old instruments could not strike it in that position. This leads me on to anotber point, which is, that a stone may be struck at one time and not at another. You will have noticed that the child had been sounded six different times at another hospital, and the stone was not felt; and when we come to look at the stone, we see that it must have been in the bladder for a verv long time. I take it that a stone of this size $\left(\frac{7}{8}\right.$ in. $\left.\times \frac{7}{8} \times \frac{1}{2}\right)$ has been forming much longer than four or five months, the period during which the symptoms have existed. Now it is absurd to suppose that in any hospital the surgeon does not know how to sound a child and detect a stone. It is a proceeding which does not require much art, and any of you would be able to do it easily enough after having passed a catheter a few times. If, then, this boy was sounded so many times and no stone detected, it could only be that for various reasons calculi, even of considerable size, cannot always be felt. The reason that it escaped notice in this instance $I$ believe to be owing to a very closely contracted condition of the bladder, and which caused a little delay in extracting the stone at the time of the operation. This was not because the stone was small, and it was not because my incision was too limited, for having once grasped the stone it came away easily; but it was because the bladder was perfectly contracted, and contained no urine. If a sound is passed into the viscus in this condition, it will have no room to play, and very likely will become entangled in the folds of mucous membrane without once reaching the surface of the stone.

In addition to the stone from which this little boy suffered, I would ask you particularly to notice another feature. He has a prepuce which is adherent to the surface of the glans. This condition is important, because it is capable of producing many most marked symptoms of calculus. Indeed I think I would go so far as to say that in nine-tenths of the children brought to you for incontinence this symptom will be owing to the adhesion between the prepuce and glans. Knowing this, and seeing that the child had such a condition, it perhaps misht have been as well to have separated the surface of these parts lefore proceeding to pass a sound. We should, however, have then had to wait to ascertain if the symptoms subsided. The boy, however, came under my notice as one in whom the presence of stone had been already settled, and therefore it was not necessary to wait; but I shall take care that circumcision is performed before he leaves the hospital to ensure his freedom from trouble hereafter; and I would advise you, whenever a case of suspected stone is brought to you, always to inspect the prepuce first, and if it be adherent, to separate it before you attempt to pass a sound.

In sounding adults whom in all probability you will, should you detect a stone, determine subsequently to litbotrise, you will find it a better plan not to do as I did in this case, for a special reason-viz., pass an ordinary sound first,but pass a lithotrite at once. It is not more difficult of introduction, and you can detect a stone with it as easily as by a sound. It drops into the bladder almost by its own weight, and you can then, should a small stone be there, if there be no indication to the contrary, crush it at once. I have pursued this plan in private practice, and only the other day a gentleman came to my house with symptoms of calculus, for whom I passed the instrument, caught the stone, and crushed it at once. The stone being broken, he was, therefore, so far on his way to being relieved of his malady. I always, where possible, use the non-fenestrated blades, that the fragments may be more completely crushed, and for convenience $I$ know of no instrument so perfect as Weiss's lithotrite, in which, by merely sliding back wards or forwards a button in the handle, the screw on the inner blade is at pleasure fixed or set perfectly free, to allow of a sliding motion on the outer blade.

I need hardly say much as to the choice of operations. You know as well as I do, that in adults, if the stone be not too large, or the bladder symptoms too intense, crushing should always be undertaken, because it is much less fatal than the cutting operation. In children, on the other hand, lithotomy is so satisfactory that one could hardly improve upon it by any other method.

I have cut a good many cases in children now, and, with the exception of a single painful case in my earlier years, all have been uniformly successful. All men have some accidents in their less skilful days of commencing practice, and I do not believe in that man who never makes any mistake. Let us only be honest, and relate them, that you who are to succeed us may be fully alive to the mishaps which may occur, and which, unless you know that similar ones have occurred before, may, when they come to you, unduly weigh upon and harass you. In one case I tell you that I failed to extract the stone, and the child died, I believe in consequence of the operation.

After lithotrity, according to the number of times you may have crushed the stone at that sitting, there will be of course more or less detritus in the bladder. The passage of this may often be much facilitated by using this instrument invented by Mr. Clover. It is most ingenious, and at times very useful. It consists of a large catheter with a very full-sized eye, or completely open at the end, to which is attached at the upper part a glass chamber, and to this, again, an india-rubber flask. The catheter is introduced into the bladder, and the air having been expelled from the flask by filling it with water, it is attached to the catheter, and the water forced into the bladder by compressing the side of the flask. When allowed to expand again the water is sucked back into the flask, and any fragments that come with it subside to the bottom of the glass chamber. This process can be repeated as often as necessary.

The prognosis in both the cases operated upon to-day is very favourable. The old man will have to be in the hospital for some time, and probably will have to undergo ten or fifteen sittings before the stone is completely pulverised and evacuated. As to this I cannot say, for sometimes there is considerable difficulty in catching the stone, and at others it passes between the blades immediately. Still, if he be long here, the risls to him is considerably less than if we performed lithotomy. In this child, on the contrary, to lithotrise would be madness. Still, though cutting is so successful in early life, no matter how skilfully performed, the operation is an operation, and as such has a certain percentage of deaths, even when undertaken by the ablest hand. The most trifling wound in certain conditions of health may conduct to death; we can hardly, therefore, claim exemption for lithotomy, which is one of the capital operations of surgery. But when I tell you that I have had forty successive cases without a death, the risk, you must acknowledge, is not so very great. The forty-first may possibly be an unsuccessful one; but, at any rate, I see no reason why it should be so at present.

\section{ON CERTAIN FATTY OR LIPOMATOUS TUMOURS AND THEIR TREATMENT BY ABSORPTION.}

\section{BY JOHN GAY, F.R.C.S., \&c.}

CASES sometimes present themselves to our notice in which a considerable number of fatty deposits, varying from ten or twenty to (it has been said) a hundred, have been met with in different parts of the body of the same person. These deposits choose, for the most part, the arms, thighs, and the buttocks, as the seats of their growth, and are by no means exclusively confined to persons otherwise more than ordinarily obese. They do not attain great size; for, as additional fatty matter is deposited, so it seems disposed rather to seek other and isolated spots in which to form itself into several small tumours than to concentrate itself in one large tumour. They are usually of firmer consistence than the ordinary solitary lipoma, and are flatter in shape, and lie more directly below, and sometimes in closer union with, the dermis. Their outline is usually, but not always, well defined; they are, as far as I am able to judge, devoid of cysts, and are more or less painful on pressure according to their proximity to cutaneous nerves. They appear to differ somewhat from the "fatty 
outgrowth" of Brodie, as well as from the "lipoma diffusum" of Lebert. These cases have not attracted much notice, in all probability from their being of comparatively rare occurrence; and $I$ cannot learn that in any one case have the tumours themselves been made the subject of post-mortem examination.

That they are composed of fat, and that the tissue does not differ from heilthy fat, with the exception of its being of firmer consistence, I cannot doubt.

One such case recently occurred in the course of my practice. I had seen others, and dismissed them with the advice that they bad better be borne with than subjected to any treatment except that suggested by Sir B. Brodieviz., the internal administration of an alkali-the liquor potassæ. The results of that treatment I have not had an opportunity of learning in any one case.

The case alluded to was that of a gentleman, over middle age, and in physique rather approaching that of the knight of La Mancha than his squire. He had noticed them for several years, but not until they had attained almost the size which they had respectively when I saw them, whilst several had become annoying from their having selected inconvenient sites, and such as had subjected them to pressure or friction. The patient's only other complaints (and these were of long standing) were slight dyspepsia, perhaps a little gout, with unusual coldness of skin and extremities, even in the warmer months of the year.

The question of treatment engaged my thoughts, and it suddenly occurred to me that, inasmuch as dead animal textures, so long as they are of normal structure and have not undergone molecular change-such as the textures used for ligature, bones, cartilage, \&c.-are removable by the absorbents, if brought into contact with them in the living body, why should these fatty tumours resist their agency under like circumstances?

The only answer that I could give to this question, having the slightest air of probability, was that the firmness of the fat in these tumours denoted a condition of their physical elements which was inimical to their absorption. It is known that the fat of these tumours is characterised by the crystallisation and separation of its elements, especially of the margarine; and it occurred to me that it might be just this change, constituting as it does an essential difference between this and ordinary living and normal fat, which is the immediate cause why the former is able to defy the action of the absorbent system. It followed consequently that it is only to liquefy the fat of such a growth or tumour by heat, and the absorbents will remove it. In accordance with this view I ordered hot baths-temperature from $120^{\circ}$ to $130^{\circ}$ if it could be borne-to be repeated as often as might be safe or convenient.* The result has been that many of these tumours have been entirely removed, whilst the nuclei of the firmer ones alone remain. I have said "the result" has been so and so. I will not venture at present to go so far without qualifying the assertion with the condition, that further experience shall concur in showing that the relation to each other of the facts narrated-viz, the treatment and the quasi cure-is more than a coincidence.

If it should turn out to be, on further evidence, a result, then it might open up the question as to the treatment of other, and what, forms of deposits through the instrumentality of the same agency.

Finsbury-place South.

\section{CLINICAL REMARKS} on

CHLOROFORM AND ITS ADMINISTRATION.

\section{BY G. VIVIAN POORE, M.D.,}

LECTURER ON MFEICAX JURISPRUDENCE AT CHARING-CROSS HOSPITAI. (Continued from page 523.)

The number of lives that chloroform has saved is certainly inestimable. The number of deaths that have been attributed to it is not more (taking the highest computation) than a few hundreds. Evil is more long-lived and makes a greater noise in the world than good; so while the

\footnotetext{
* The mode of application of the heat is of course open to variation.
}

fact of a life saved by chloroform makes no noise beyond the small circle of acquaintance of the owner of said life, a death from chloroform is considered food for hungry penny-a-liners, and monopolises a paragraph in every country newspaper.

On account of the notoriety which attaches to it, as well as on account of its intrinsic awfulness, death from chloroform has largely attracted the attention of scientific men. It behoves us, therefore, carefully to review this matter, and ask ourselves in how many ways chloroform may kill; or, perbaps, a more practical form of putting the question would be, - In how many ways may death take place during an operation under chloroform?

There is no doubt that chloroform is not answerable for all the deaths that have been attributed to it. Sudden deaths on the operating table were by no means unheard of before the days of chloroform. Sir James Simpson has collected and published ten such cases, in which the shock of an operation, reacting upon a heart already weakened by debility and fright, occasioned fatal syncope. The same thing may occur it the patient be only partly anæsthetised. Professor Lister mentions a case in which amputation of the penis was to be performed, and in which, the patient not being completely anæsthetised because the operation was short, cessation of the pulse and death were coincident with the single stroke of the knife necessary for the operation. This he maintains could not have occurred if the patient had been fully narcotised, for then it would have been impossible for the shock of the operation to have reacted on the heart. (Or, if the heart had not been weakened by chloroform, would the shock of the operation have proved fatal to it?) Death may also occur from loss of blood during the operation. There is no donbt that a good many of the fatal cases of chloroform have in reality been cases of syncope resulting from one or other of these causes, the chloroform itself playing only a small, or perhaps not any, part in the fatal result. There are however cases, and many such, in which death is due solely to the employment of chloroform, and to such cases I wish now particularly to direct your attention.

Death from chloroform may occur in two ways:-1. Suddenly; the pulse ceasing either before or together with the respiration. 2. Gradually; the respiration becoming embarrassed before any effect is produced on the pulse. Death in either of these ways is brought about by an overdose of chloroform. In the first case, by the quick administration of a highly concentrated chloroform atmosphere; and in the second case, by the long-continued administration of a moderate dose. Dr. Snow and others have proved that the main danger is in giving a too concentrated chloroform atmosphere. The committee of the Medico-Chirurgical Society proved beyond a doubt that chioroform very soon weakens the heart; and they invariably found that the pressure on the femoral artery of dogs and other animals, as measured by the hæmadynamometer, got gradually less and less as the animal succumbed to the influence of chloroform. The heart therefore (more especially if it be in a condition of fatty degeneration) is, one might say, in all cases of chloroformisation prone to stop at slight causes.

I will first endeavour to explain-although the matter is not capable of rigid proof-how a strong dose of chloroform causes sudden stoppage of the heart. It is not, I think, reasonable to suppose that in these cases the stoppage of the heart is due to the excess of chloroform which has found its way into the muscular substance of the heart. It is unreasonable, it seems to me, to assert that an atmosphere of chloroform of a certain strength, which we will designate by the number 6 , inhaled only for a few moments, will cause more chloroformisation of the heart itself and of the symparhetic nerve than an atmosphere of 4 inhaled for an indefinite period. This theory is unreasonable, and to my mind carries no conviction. Dr. Anstie explains the different modes of death in this way. He says: "When the impregnation of the blood takes place with moderate rapidity, the sympathetic nervous system is the ultimum moriens, and death begins at the lungs; but when, on the contrary, the circulation becomes very rapidly impregnated with a large proportion of chloroform, the narcotic effect may fall with such force on the sympathetic nerves as to extinguish their vitality at once.

I would explain the mode of death from chloroform by its action on the pneumogastric nerve. It is a matter of every. 